\title{
DOR PÓS OPERATÓRIA EM TRATAMENTOS ENDODÔNTICOS EM SESSÃO ÚNICA: REVISÃO DE LITERATURA
}

\author{
João Eduardo Nedel dos Reis ${ }^{\text {; }}$ Flávia Kolling Marquezan 2; Janice Almerinda \\ Marin ${ }^{3}$
}

\section{RESUMO}

O tratamento endodôntico visa promover um ambiente compatível com saúde ou reparo dos tecidos periapicais.Tradicionalmente é realizado em várias sessões, utilizando uma medicação intracanal entre o preparo químico-mecânico e a obturação, a fim de eliminar microrganismos e seus subprodutos do sistema de canais radiculares antes da obturação bem como reduzir o desconforto ao paciente. Sendo assim, o presente trabalho tem como objetivo avaliar, por meio de uma revisão de literatura, a dor pós operatória em tratamentos endodônticos realizados numa única ou múltiplas sessões. Para isso revisou-se a literatura na base de dados das seguintes plataformas virtuais: PubMed, SciELO, Medline e Cochrane Library utilizando as seguintes palavras-chave indexadas noDeCS: "Endodontics", "Pain" e "Postoperative". Dentre os critérios de elegibilidade estão revisões sistemáticas e ensaios clínicos. Após a busca, foram selecionados 22 artigos sendo incluidos 6 na revisão. Com a análise dos estudos, concluiu-se que a dor pós-operatória apresentouresultados semelhantes entre o tratamento endodôntico realizado em uma ou múltiplas sessões.

Palavras-chave: Dor; Sessão Múltipla; Tratamento de Canal.

Eixo Temático: Atenção Integral e Promoção à Saúde.

\section{INTRODUÇÃO}

O tratamento endodôntico objetiva promover um ambiente compatível com saúde e reparo dos tecidos periapicais logo após intervenção terapêutica, tornando possível o retorno de suas funções. Para que isso aconteça, é necessário fazer a limpeza e modelagem dos condutos para desinfecção dos sistemas de canais radiculares, obturação e selamento coronário (WONG et al., 2014).

A ocorrência da dor após o tratamento endodôntico ocorre devido à resposta

\footnotetext{
1 Odontologia/UFN, Santa Maria - RS/ joao.reis@ufn.edu.br

2 Odontologia/UFN, Santa Maria - RS/ jmarin@ufn.edu.br

${ }^{3}$ Odontologia/UFN, Santa Maria - RS/ flavia.marquezan@ufn.edu.br
} 
inflamatória aguda nos tecidos periapicais, podendo iniciar dentro de poucas horas ou dias após o tratamento. Muitas vezes, o paciente considera o desconforto um indicador de ineficiência do cirurgião dentista, comprometendo a confiança no profissional ou a satisfação perante o tratamento. Os fatores etiológicos da manifestação da dor não são determinados com precisão. No entanto, inúmeros fatores químicos, mecânicos e/ou microbianos nos tecidos periapicais podem estar envolvidos. O aparecimento da dor depende da intensidade dos danos aos tecidos e - resultado do tratamento é influenciado pela persistência da sintomatologia dolorosa (SU, Y. et al., 2011).

Ao investigar a dor pós tratamento endodôntico, os estudos não encontraram diferença estatisticamente significante, tanto no que se refere ao número de sessões (uma ou múltiplas) (EL MUBARAK et al., 2010; RIAZ, A et al., 2018) ou nas diferentes situações, polpas vitais e necrosadas (ALOMAYM et al., 2019). Na revisão sistemática de ensaios clínicos, randomizados ou quase randomizados, SU et al. (2011) constataram uma prevalência de dor nas primeiras $72 \mathrm{~h}$ em $26 \%$ dos pacientes tratados numa sessão e em $37 \%$ daqueles tratados em múltiplas sessões.

Esses achados corroboram com outra revisão sistemática de ECR, ROSSO, C et al., 2012 verificaram a presença de dor pós operatória em dentes com infecções que receberam a terapia endodôntica em sessão única e terapias em múltiplas sessões, os autores conseguiram incluir apenas dois artigos pela heterogeneidade das publicações e obtiveram como resultado menor dor pós operatória para múltiplas sessões com uso de hidróxido de cálcio quando os dentes já apresentavam dor pré-existente. Nos casos assintomáticos, a presença de dor foi semelhante em ambos os tratamentos uma ou múltiplas sessões. Dada a relevância no assunto bem como a vasta literatura, o objetivo deste trabalho é avaliar, por meio de uma revisão de literatura, a dor pós-operatória em tratamentos endodônticos realizados numa única ou múltiplas sessões, auxiliando o profissional na melhor escolha para o seu paciente. 


\section{METODOLOGIA}

O presente estudo consistiu em uma revisão narrativa de literatura. $O$ levantamento bibliográfico foi realizado nas base de dados : PubMed, SciELO, Medline e Cochrane Library utilizando os seguintes termos indexados noDeCS: "Endodontics", "Root Canal Therapy", "Pain" e "Postoperative". Dentre os critérios de elegibilidade, estão o idioma, ano de publicação e delineamento do estudo, sendo considerados elegíveis os artigos em português e inglês, publicados nos últimos 10 anos e delineado revisões sistemáticas, de literatura e ensaios clínicos. Foram excluídos artigos não encontrados na íntegra, fora do delineamento elegível ou com amostra representativa de crianças. As buscas foram realizadas no período de 2021, por um examinador experiente no assunto.

\section{RESULTADOS E DISCUSSÕES}

Os resultados desta revisão foram explorados e apresentados no quadro 1. Observa-se a grande diferença na metodologia dos artigos, especialmente no que se refere aos tempos de avaliação da dor pós-operatória, variando de algumas horas até alguns meses. Períodos de 6, 12, 24 e 48 horas (ALOMAYM, M. et al., 2019; 12 e 24 horas (EL MUBARAK, A.; ABU-BAKR, N.; IBRAHIM Y.E., 2010.); 48 horas (RIAZ, A. et al., 2018); 72 horas e 1 semana (ROSSO, C. et al., 2014). 24 horas a 30 dias (SU, Y. et al. 2011) 2 dias a 2 meses (WONG, A.; ZHANG, C.; CHU, C.H., 2014).

Além disso, a maioria dos artigos utiliza uma escala analógica de dor, podendo ser considerada uma limitação frente a subjetividade da percepção de dor do indivíduo pesquisado (ALOMAYM, M. et al.2019, EL MUBARAK, A.; ABU-BAKR, N.; IBRAHIM YE. 2010 e RIAZ, A. et al. 2018).

As condições da polpa, vitais ou não vitais, estão diretamente adjuntas a possibilidade de infecção e dor da região apical, visto que pode ser associadas a reinfecção ou infecção da região apical. Polpas não vitais foram analisadas por RIAZ, A. et al. 2018; ROSSO, C. et al. 2014; SU, Y. et al. 2011; e a comparação de polpas vitais e não vitais foram avaliadas nos artigos de ALOMAYM, M. et al.2019; EL MUBARAK, A.; ABU-BAKR, N.; IBRAHIM YE. 2010.

Quadro 1: Resultados 


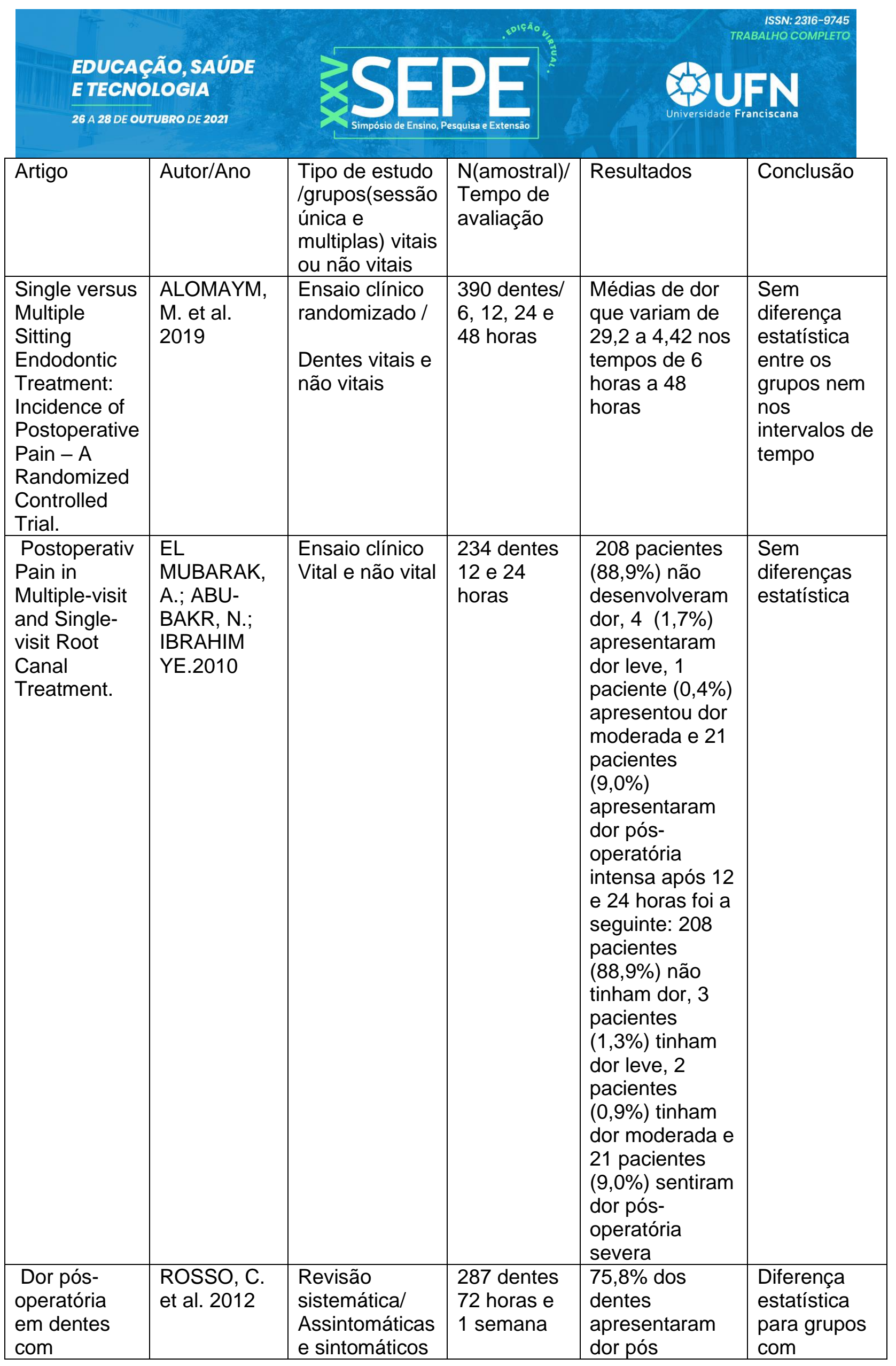




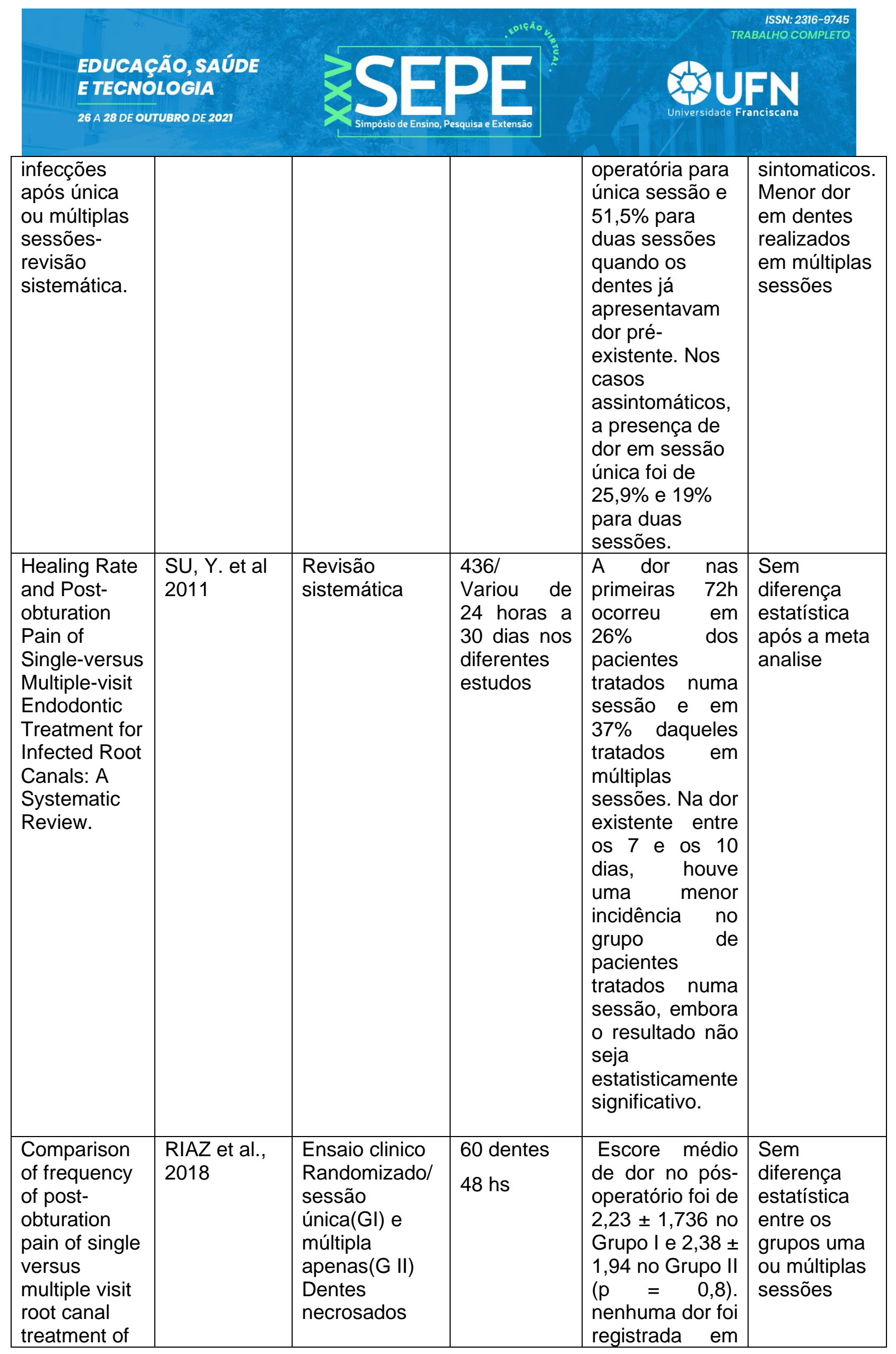




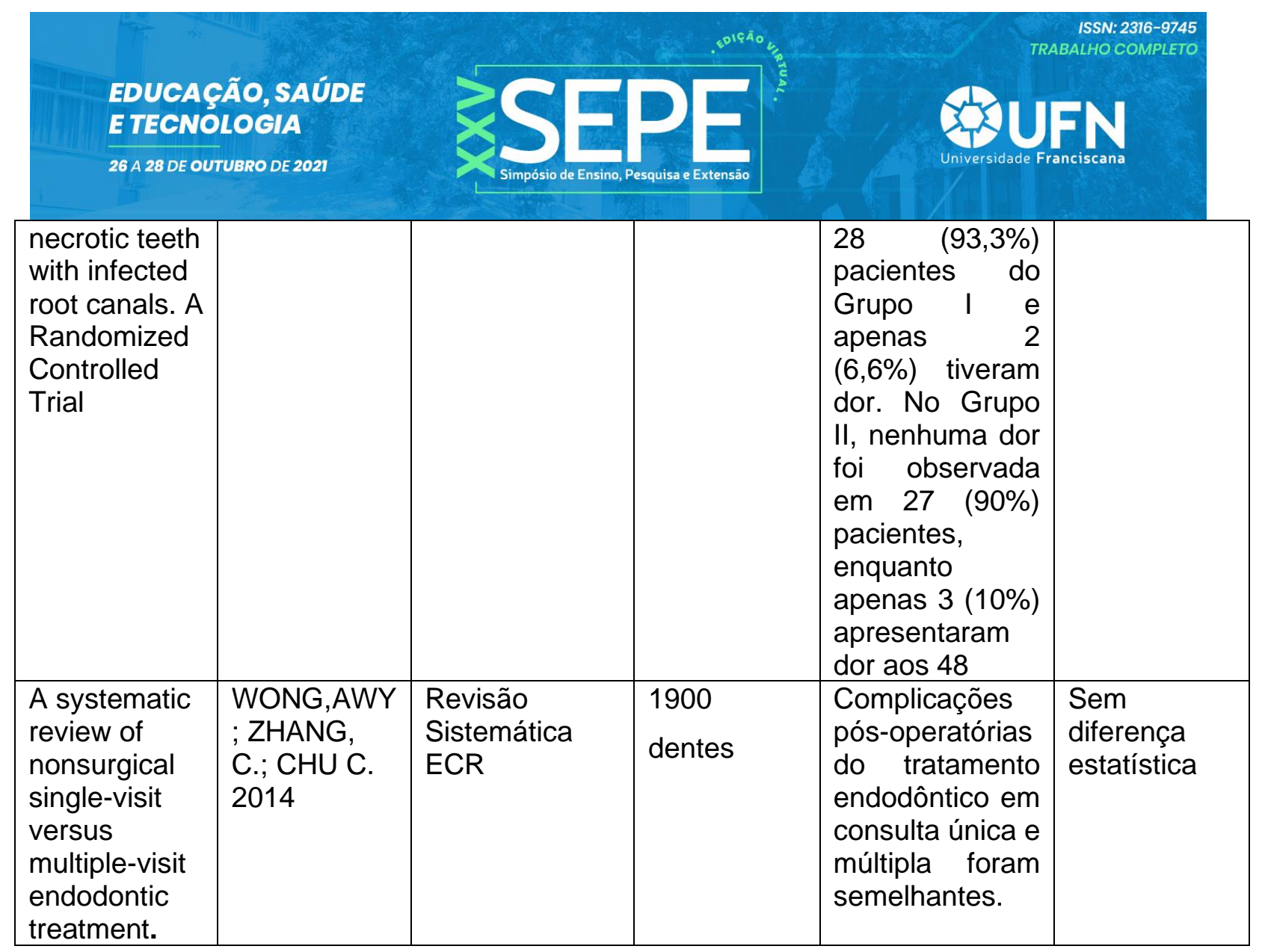

Fonte: Elaborado pelo autor, 2021

\section{CONCLUSÃO}

Foi observada uma diferença estatisticamente não significante entre os valores de dor pré-operatória e pós-operatória dos dentes vitais e não vitais entre uma e multiplas sessões na terapia endodôntica

No que se refere a tratamentos endodônticos realizados em dentes que inicialmente apresentavam sintomatologia dolorosa, quando realizados em uma única sessão apresentaram maior indice de dor pós operatória. Já em dentes assintomáticos, não houve diferença de relatos de dor pós operatória independente do número de sessões

Sendo assim, podemos constatar que em casos assintomáticos rotineiros na prática clínica, não há diferença quanto ao número de sessões. Entretanto, há a necessidade de ensaios clinicos bem delineados com tempos de avaliação padronizados para que a comparação entre os artigos possa ser passivel de ser realizada. 


\section{QUFN}

\section{REFERÊNCIAS}

ALOMAYM, M. et al. Single versus MultipleSittingEndodonticTreatment: IncidenceofPostoperativePain - A Randomized Controlled Trial. Journal of International Society of Preventive \& Community Dentistry, vol. 9, n. 2, p. $172-$ 177, 2019.

EL MUBARAK, A.; ABU-BAKR, N.; IBRAHIM YE. Postoperativ Pain in Multiple-visit and Single-visit Root Canal Treatment. Journal of Endodontics, vol. 36, n. 1, p. 36$39,2010$.

RIAZ, A. et al.Comparison of frequency of post-obturation pain of single versus multiple visit root canal treatment of necrotic teeth with infected root canals. A Randomized Controlled Trial. Journal of the Pakistan Medical Association. Vol. 68, n 10, p. 1429-1433, 2018.

ROSSO, C. et al. Dor pós-operatória em dentes com infeções após única ou múltiplas sessões- revisão sistemática. Pesquisa Brasileira em Odontopediatria e Clínica Integrada João Pessoa, vol. 12, n.1, p.143-48, 2014.

SU, Y. et al. Healing Rate and Post-obturation Pain of Single-versus Multiple-visit Endodontic Treatment for Infected Root Canals: A Systematic Review. Journal of Endodontics, vol. 37, n.2, p. 125-132, 2011.

WONG, A.; ZHANG, C.; CHU, CH. A systematic review ofnonsurgical single-visit versus multiple-visitendodontictreatment. Dove Press Journal:

ClinicalCosmeticandlnvestigationalDentistry, vol. 6, n. 6, p. 45-56, 2014. 